\title{
TECHNIQUES FOR ROAD TRAFFIC OPTIMIZATION: AN OVERVIEW
}

\author{
Ahmad AA Alkhatib \\ Computer Information System, Alzaytoonah University of Jordan, \\ Airport Street, Amman, 11733, Jordan \\ Ahmad.Alkhatib@Zuj.edu.jo \\ Thaer Sawalha \\ Computer Science, Alzaytoonah University of Jordan, \\ Airport Street, Amman, 11733, Jordan \\ Sawalha1992@gmail.com
}

\begin{abstract}
With the number of cars clogging the roads and junctions around the world and expected to double in the next decades, new ways have been introduced by researchers. controlling traffic signals through active traffic management targeted to increase road capacity and road traffic flows more efficient. Smart traffic lights have the ability to reduce traffic congestion and vehicle waiting time, thus reducing fuel wasting and gas emissions. This work explores proposed solutions and techniques for traffic signal to optimize road traffic and reduce traffic congestion impacts.
\end{abstract}

Keywords: Road Traffic Optimization, Traffic Light, Traffic Signal, Genetic Algorithm, Fussy Logic, VTL, Neural Network, V2V, V2I, V2N

\section{Introduction}

Traffic congestion has been causing many challenges in crowded cities. congestion means lost time, fuel, and frustration.

The problem summarized with the significant increasing number of vehicles every year in all parts of the world, especially in major cities, leads to acute traffic jams appeared [1], therefore, the need to create a new infrastructure such as bridges, tunnels or traffic lights at intersections is necessary to facilitate the flow of vehicles. Bridges and tunnels is not always the perfect solution due to the high cost of construction, maintenance and the lack of space. On the other hand, the use of traffic light signals with normal fixed cycles will increase traffic jams and carbon dioxide emissions [2, 3, 4].

Therefore, researchers recently worked on proposing several techniques that manage intersections in a smart way that is appropriate to the actual current of vehicles flow. In this paper, some of these suggested techniques used to manage intersections in a smart way are presented.

\section{Techniques Overview}

Traffic jams and waiting time is a very crucial problem in major cities, where Improving traffic at intersections, will help in reducing traffic congestion, vehicle travel time, and carbon dioxide emission in the atmosphere.

Traffic optimization methods to improve the flow of vehicles at intersections can be divided into two main parts based on the infrastructure used to implement them:

\subsection{Intelligent Traffic Light System}

$$
\begin{aligned}
& \text { * Intelligent Traffic Light System. } \\
& \text { * Virtual Traffic Light System. }
\end{aligned}
$$

There are many methods that have been used in an attempt to optimize vehicles traffic flow at intersections such as: the genetic algorithm $[1,5]$, neural networks $[6,7,8]$, controller $[9,10]$ and fuzzy logic $[11,12]$ etc. Below is an explanation of some of them:

\subsubsection{Genetic Algorithm}

Genetic algorithm is a type of optimization algorithm based on the theory of natural selection and evolutionary biology. Genetic algorithm can be used to find a solution to control traffic flow at intersections. It has the ability to constantly renew decisions and create solutions [1].

Genetic algorithm is implemented in five stages starting with; initial population, fitness function, selections, crossover, and mutation [1]. See figure 1 


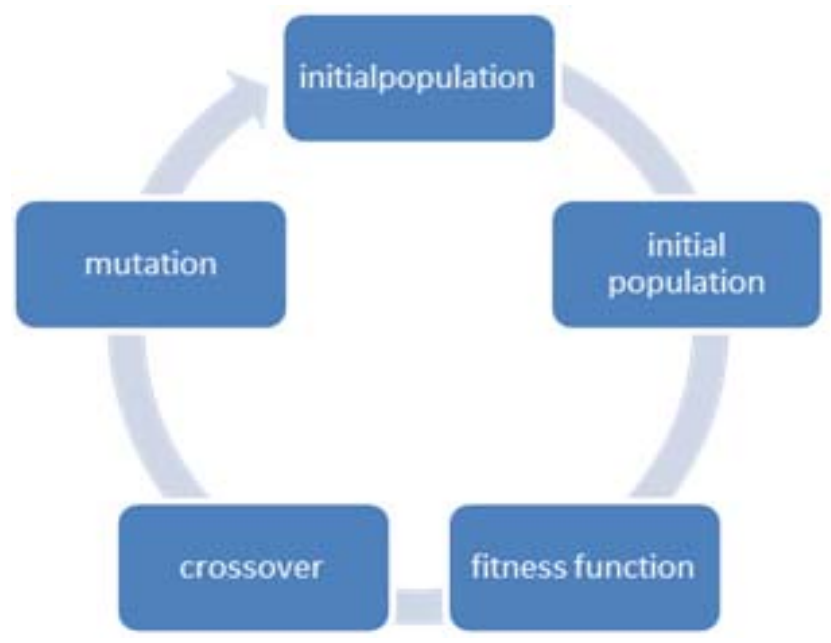

Fig. 1. Genetic Algorithm Stages.

Singh et al [5] proposed using the genetic algorithm to improve the flow traffic of vehicles at intersections, as they worked to increase the time of the green light up to five seconds, which showed improvement results by $21.9 \%$.

Tamimi et al [1] proposed genetic algorithm applied to one intersection and four traffic lights. In their work, they suggested a minimum green time of 10 seconds and a maximum green time of 60 seconds. The results showed after Matlab development simulation a $28.4 \%$ improvement in the number of vehicles that left the intersection compared to other works using the same algorithm.

Genetic algorithm disadvantages summarized in:

$\checkmark$ Delay of decision

$\checkmark$ Population generation

$\checkmark$ Such complex method requires high resources

\subsubsection{Fuzzy Logic}

Fuzzy logic is a way to deal with registering dependent on "degrees of truth" as opposed to the standard "true or false" or ( 1 or 0$)$ Boolean rationale in which the cutting edge PC is based. Fuzzy logic works closer to the way human minds work. It processes information gathered in real time to get results that are relevant to current changes [11]. See Figure 2.
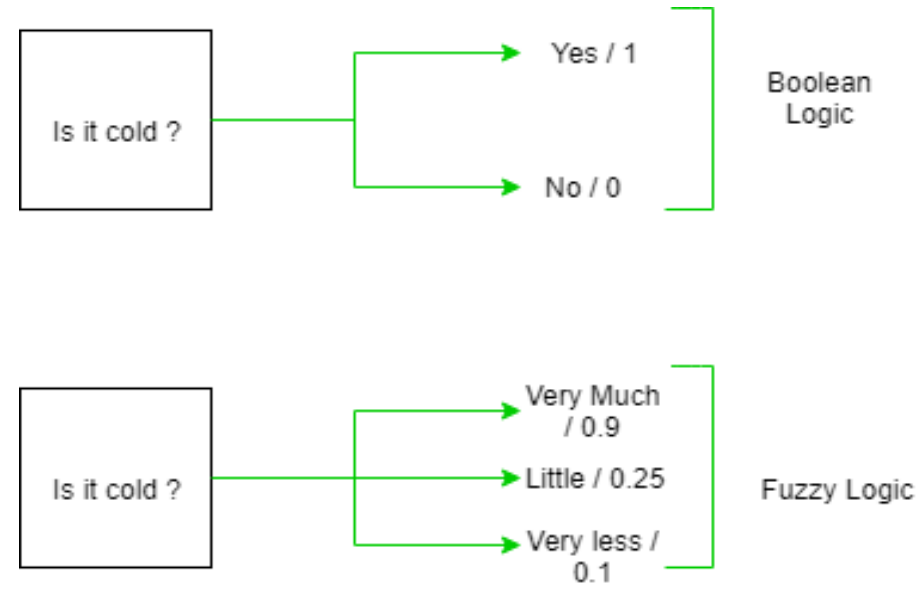

Fig. 2. The Difference between Boolean logic and fuzzy logic [12]

Fuzzy logic has been used in several proposals to improve the flow of vehicles, for example, a suggestion Omina [13] has adopted a cross-sectional study aimed at monitoring traffic in the city of the central business district of Nairobi and its surrounding areas. The data was collected through observations about traffic behavior at three intersections, namely Railways, Hili Salice and the General Post Office. The data was analyzed and presented using descriptive statistics; tables and graphs using excel 2003. To test the adaptive traffic signal control, a simulation using MATLAB tools with some development using $\mathrm{C}++$ and Qt software for validation. The results showed that the use of the fuzzy logic was successful as it reduced the average vehicle waiting time compared to the fixed control units. See figure3 


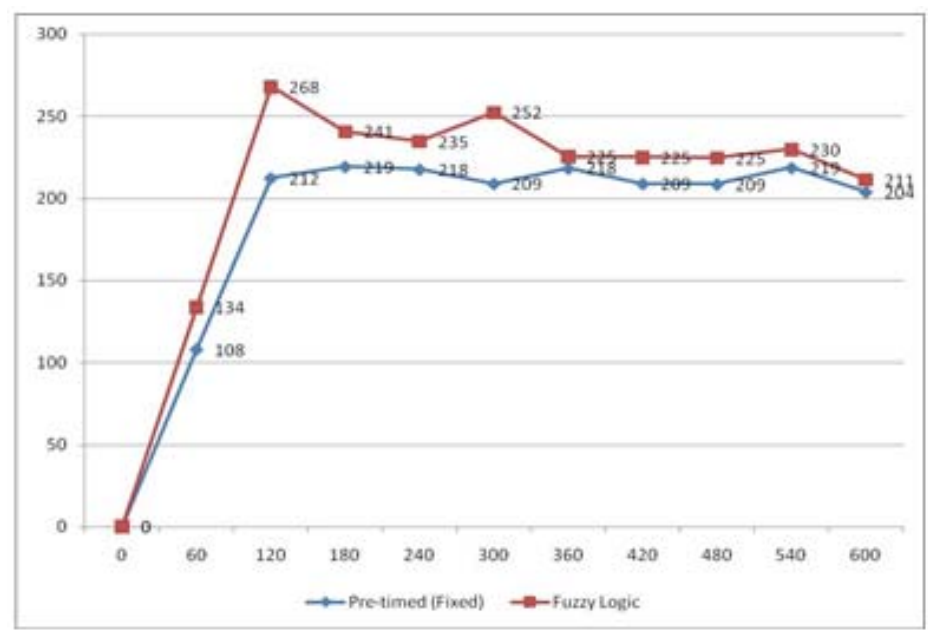

Fig. 3. The Frequency Performance Chart [13]

\subsubsection{Neural Network}

The neural network is located within one of the types of data modeling. It is nonlinear algorithm with adaptive properties. Neural networks consist of three layers which are divided as follows: the input layer, the hidden layer, and the output layer [6].

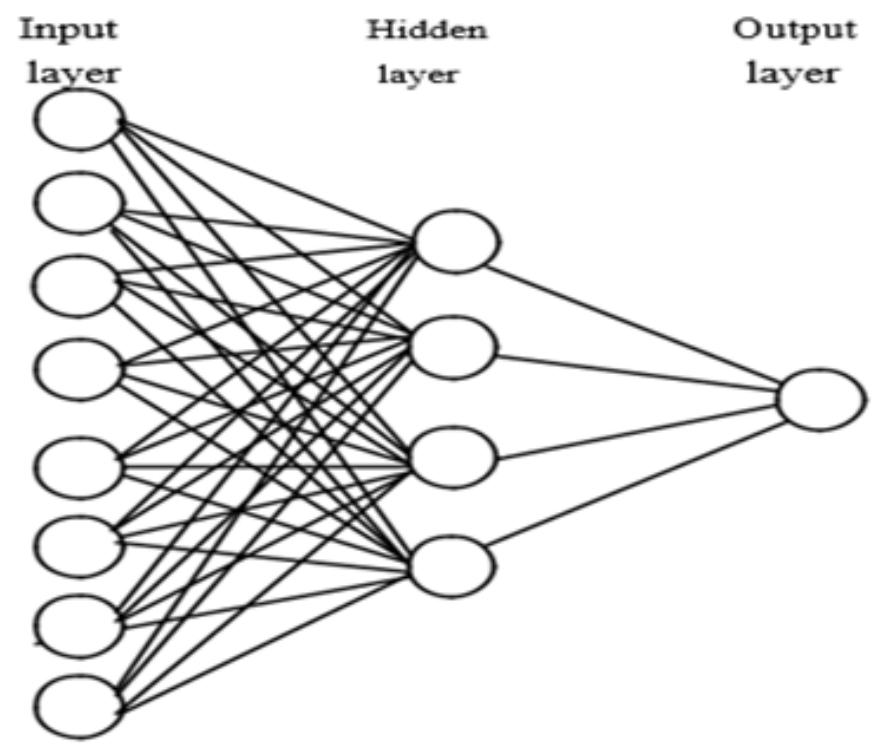

Fig. 4. Simple neural network architecture

Researchers suggested using the neural network in the field of traffic, where one of the proposed work presented by Fazli et al [7] using camera installed at an altitude almost near the road surface to discover and classify the vehicles. At beginning the photos are taken of the passing vehicles and then remove the appendages from the image, then vehicles are classified according to the image taken in three categories: heavy vehicles, light vehicles and motorcycles in the second stage.

On the other hand, Chao et al [8] Improved the traffic flow of vehicles using extension neural network (ENN) at intersections. The proposed algorithm worked to determine the number of vehicles and calculate the time required by one vehicle to cross the intersection in the case of the green light for each of the main road and subline, then the results are used to calculate the green light time for the next cycle. 


\subsubsection{Microcontroller And Programmable Logic Controller (PLC)}

A programmable logic controller and controller were used to improve the flow of vehicles at intersections in different ways.

Viswanathan,and Santhanam [9] suggested wireless sensor networks to predict the movement of vehicles near intersections, where the sensors were distributed at four levels to collect information about the density of vehicles on the road. Density of vehicles in the intersection is determined based on the sensor readings sent over to a controller using the ZigBee protocol. The controller uses the sensor data in routing algorithm to control the intersection management.

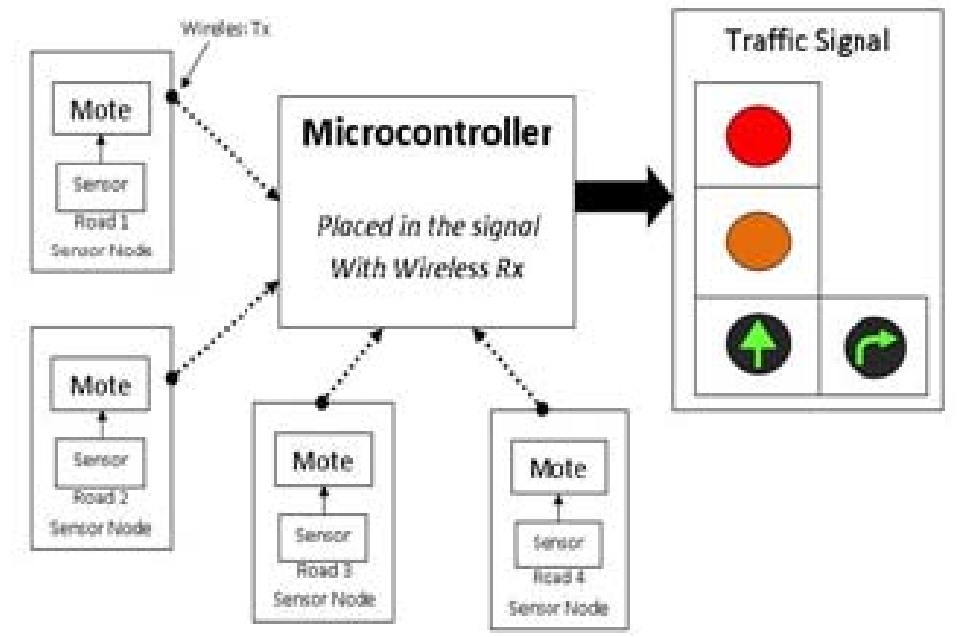

Fig. 5. Block Diagram of the Proposed System [9]

Viraktamath et al [10] suggested using the Indra Logic L20 PLC as a central unit to implement the system, as the sensors were distributed over the lane near the intersection to supply the PLC with the number of vehicles in the intersection to help them make the appropriate decision to manage the intersection.

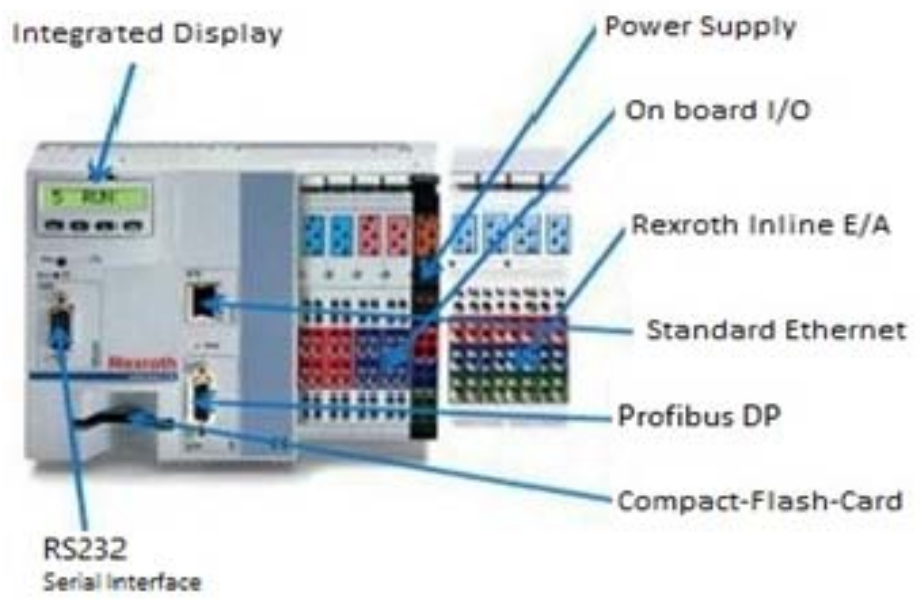

Fig. 6. Indra Logic L20 PLC [10]

\subsection{Virtual Traffic Light System}

Despite the improvements made by using the intelligent traffic signal, there are some challenges that have not been addressed, such as the cost of installation of traffic signals on intersection and the high cost of operation and maintenance. Therefore, researchers have directed at the present time of smart cities, IoT, Vehicle-toeverything (V2X) work on developing the traffic light using the virtual traffic light (VTL) appears on the vehicle screen. 


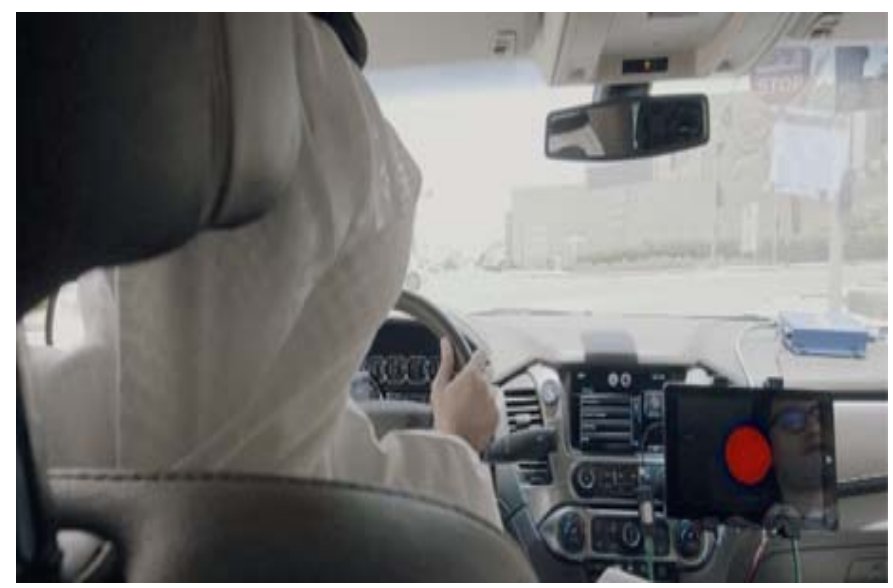

Fig. 7. Vehicle Screen

Virtual Traffic Lights (VTL) was initially established in 2010. They aim to solve the problem of the traffic jam by using communications paradigms that can be divided into four main sections: Vehicle-to-Vehicle (V2V) and Vehicle-to-Infrastructure (V2I) and Vehicle-to-Network (V2N) and Vehicle-to-Pedestrian (V2P) [14]. see figure 8. Where it is used for connection in communications paradigms Dedicated Short Range Communications (DSRC) at a frequency of $5.9 \mathrm{GHz}$, in addition to using cellular and wireless technologies $2 \mathrm{G}, 3 \mathrm{G}, 4 \mathrm{G}[15]$. Some of the VTL goals are listed in the figure 9.

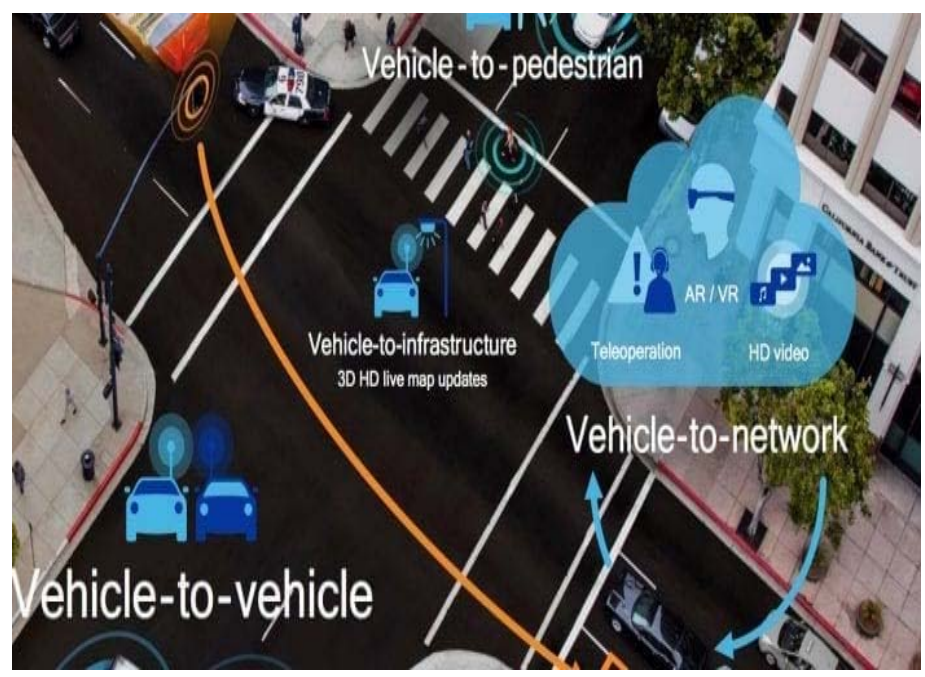

Fig. 8. Communications Paradigms [16] 


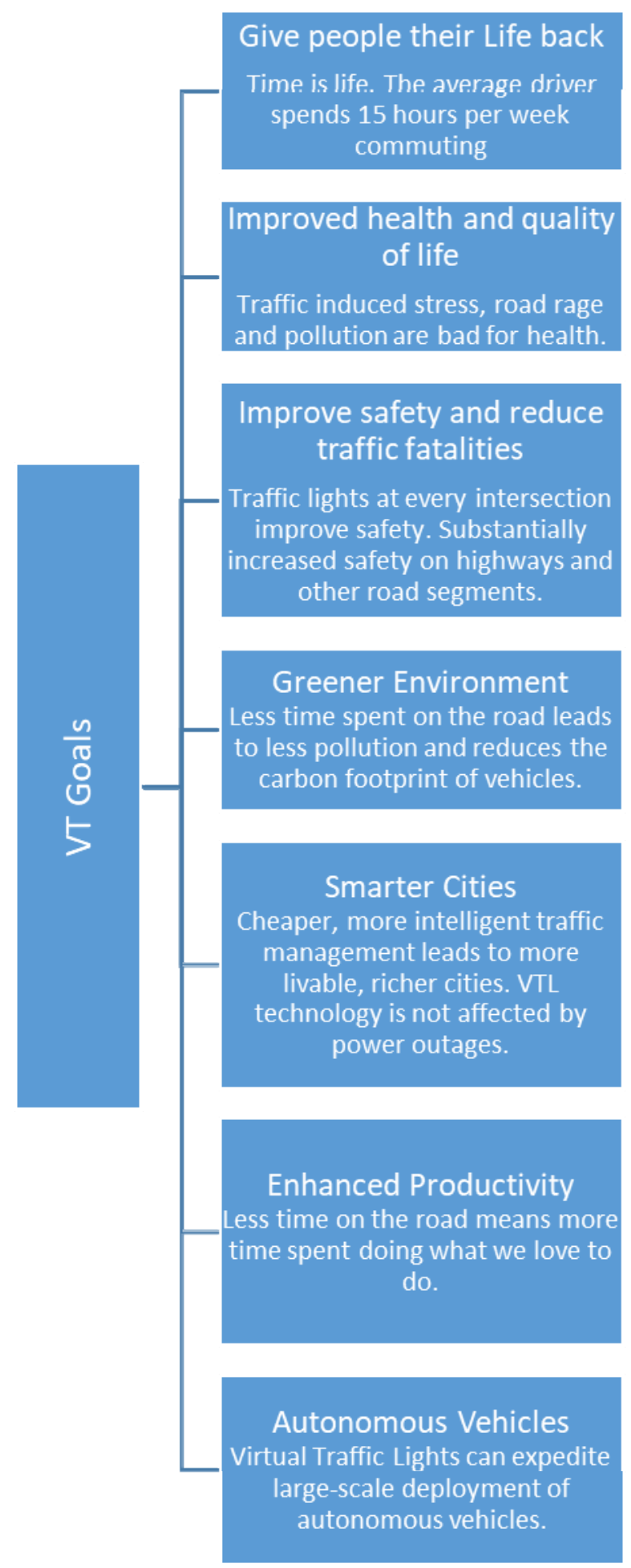

Fig. 9. VTL Goals

In terms of applications, the three pillars of VTL technology are [17]:

$>$ Traffic efficiency, where the early experiments results showed that the use of VTL improved the flow of vehicles in intersections.

$>$ Traffic Safety, VTL improves road safety, as all intersections can be covered by traffic without the need for expensive infrastructure and operating, in addition to seeing the traffic light inside the vehicle clearly away from bad weather such as fog.

$>$ Autonomous driving, the use of VTL will help to connect vehicles to each other and thus each vehicle will have the ability to communicate with neighboring vehicles and make priority decisions on the roads without the need for a driver. 


\subsubsection{Vehicle-To-Vehicle (V2V)}

Many researchers worked on the technology of transfer from vehicle to vehicle (V2V) [18, 19, 20] to improve the flow of vehicles at intersections, where the results of the experiment showed the effectiveness of this technology, as this technology dispenses the infrastructure for each intersection to be replaced by one of the vehicles in the intersection called leader Which is chosen based on a specific algorithm in turn to manage other vehicles in intersection. See figure 10.

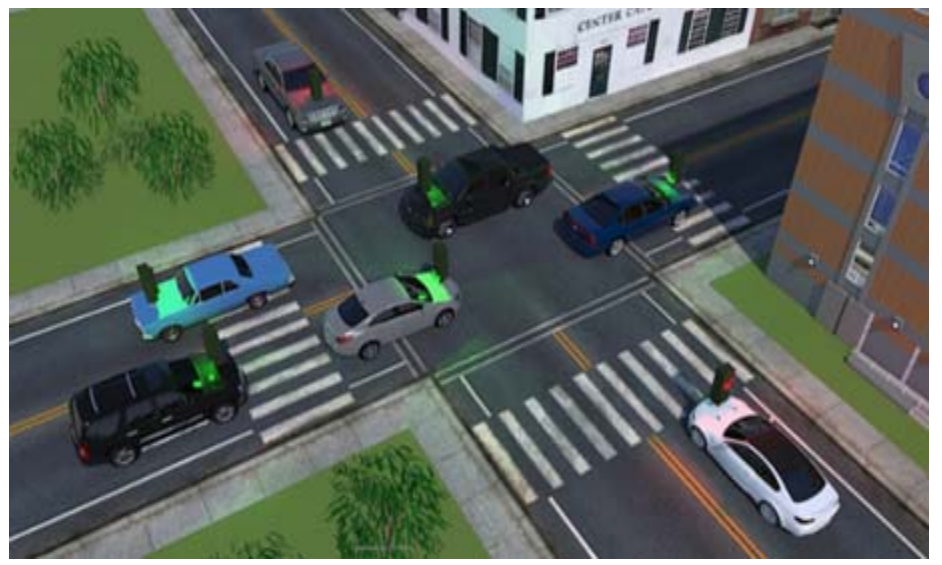

Fig. 10.V2V communication

For example: Zhang et al proposed. [18] A prototype system designed using VTL technology as a potential solution to reduce traffic congestion during rush hours and out of rush hours, the work of the model was based on the use of using Dedicated Short Range Communications (DSRC) technology for communication the VTL.

The principle of the VTL process was adopted as follows:

1) Sensing: Vehicles approaching an intersection detect other vehicles approaching the same intersection.

2) Leader Election: Vehicles choose a leader vehicle that acts as a traffic light.

3) Broadcast: The leader vehicle publishes traffic signal information to other vehicles on the intersection.

4) Handover: When the leader vehicle wants to exit from the intersection, the leader vehicle will deliver its driving role to one of the other vehicles in the intersection.

5) Release: When the leader vehicle does not detect any opposing vehicle, it will terminate the VTL.

They performed testing in an empty parking area, where the proposed model was applied in the field of two vehicles, the results showed that the use of the VTL improves $20 \%$ of the total travel time compared to the use of stop signs at each intersection.

Ferreira et al. [21] suggested moving from traditional signals to virtual traffic light inside the vehicle as it is supported by communications vehicle-to-vehicle (V2V).

They assumed that all vehicles are equipped with DSRC devices, and all vehicles share the same digital road map, and all vehicles are equipped with a global positioning system (GPS), and finally, they assumed that the wireless protocol is safe and reliable and its efficiency is appropriate to the requirements of VTL.

Each vehicle is provided with a dedicated application unit (AU) where intersection information is saved in advance to determine the locations of intersections that need to create a VTL. When the vehicle approaches these intersections, the AU searches for a VTL signal followed by, if not found a new VTL will be created.

If there is a need to create a VTL, the vehicles that approach the intersection will choose a vehicle to be the leader (the vehicle closest to the intersection) The leader vehicle will create and manage the VTL, where it will send traffic directions to facilitate the flow of vehicles temporarily, and then hand over the intersection management to another vehicle In view with it to complete the process of managing the intersection and maintaining the VTL, and so on until the vehicles do not remain inside the intersection so that the VTL is terminated.

The proposed model was applied using DIVERT simulation, where the results showed an improvement in vehicles flow with an increase of more than $60 \%$ in the intersection.

(V2V) communication disadvantages summarized in:

$\checkmark \quad$ Not effective at high traffic density.

$\checkmark \quad$ Actual and historical data cannot be combined at the vehicle level. 


\subsubsection{Vehicle-To-Network (V2N)}

Other proposed technique uses virtual traffic light (VTL) technology is vehicle-to-Network (V2N), here are some suggestions based on this technology. See Figure 11

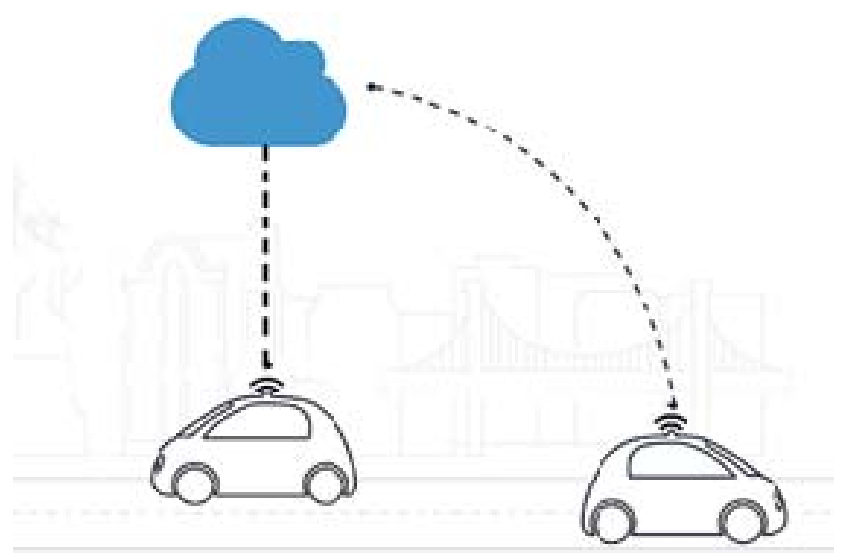

Fig. 11. V2N Communication

Münst et al. [22] proposed a VTL system by utilization of mobile communication technologies to send data from vehicles to cloud computing. Data is processed to provide decision in a real-time for vehicles to go or stop at intersections.

The basic data flow encompasses the following operations:

1) The vehicle send data to the cloud computing by the TMP protocol;

2) The cloud application programming interface (API) receives data for processing.

3) The Kalman filter removes the outliers.

4) The "map matching" algorithm and filter Kalman work together to determine the locations with the highest accuracy, then the output of the curing process is stored in HBase.

5) After adding the vehicle data collected in real time with the previous data, it is sent to the traffic control application.

6) The traffic controller determines priorities and broadcasts speed recommendation messages via Temp Protocol. See Figure 12.

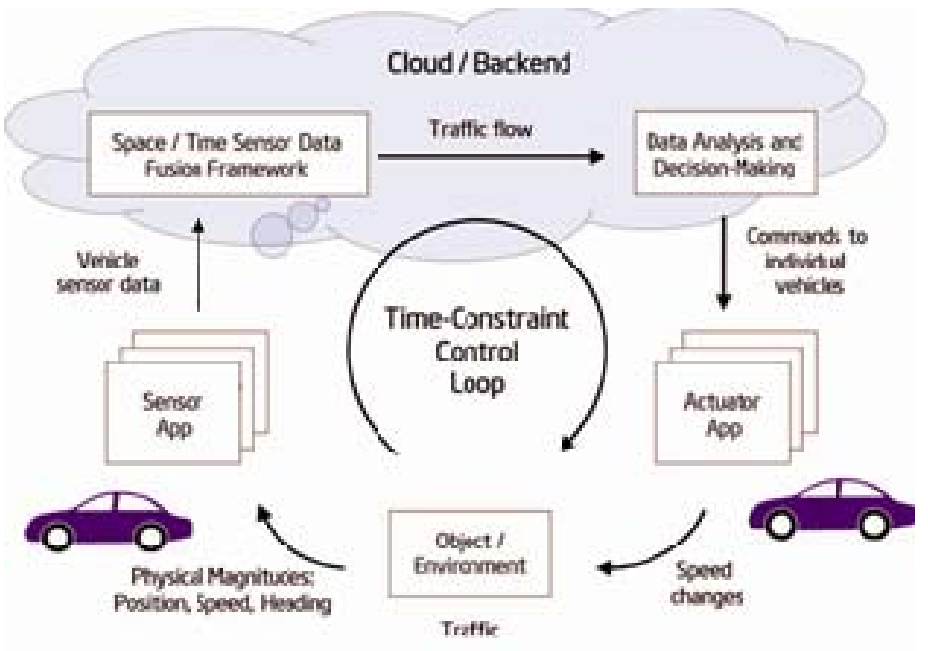

Fig. 1.V2N communication [22]

One of the determinants that has emerged is the decreasing effectiveness of the system as the number of human operator's increases. 


\subsubsection{Vehicle-to-Infrastructure (V2I)}

V2I technology based on simple infrastructure is used at each intersection to control the flow of vehicles and prioritize them, for example;

Al-qutwani and Wang [23], proposed A new model for intersection management relied on Named Data Networking (NDN). NDN sends and receives data based on the content name and not the content title, where they used the roadside unit (RSU) to act as the intersection controller instead of the traffic light and then RSU collects vehicle information that have reached or will arrive at the intersection to be processed according to the priority policy. Then RSU sends an instant message to each vehicle that allows it to continue on the road or wait for a period of time.

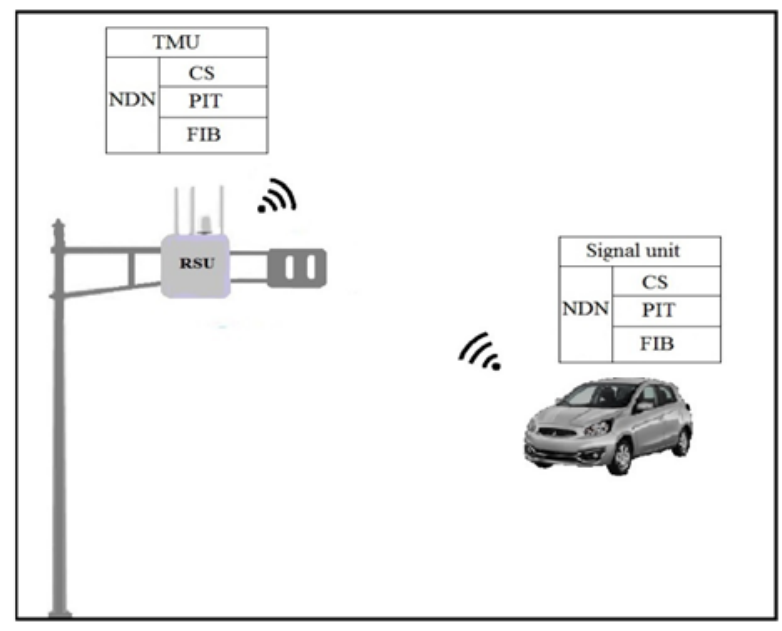

Fig. 12. V2I communication [21].

\section{Conclusion}

In this paper we discuss different techniques for traffic light optimization in both technologies: Intelligent Traffic Light and Virtual Traffic Light. The most common solutions are genetic algorithm, fussy logic, neural network and VTL with all different networking option V2V, V2N and V2I.

Genetic algorithm, fussy logic and neural network require high resources for computation and processing. On the other hand, VTL is the main trends in road traffic optimization since it does not require expensive physical traffic signals and it can adopt all the classic and new techniques used in traffic signal control

\section{References}

[1] Tamimi, A., AbuNaser, M., Tawalbeh, A. A., \& Saleh, K. (2019, April). Intelligent Traffic Light Based On Genetic Algorithm. In 2019 IEEE Jordan International Joint Conference on Electrical Engineering and Information Technology (JEEIT) (pp. 851-854). IEEE.

[2] Ferreira, M., \& d'Orey, P. M. (2011). On the impact of virtual traffic lights on carbon emissions mitigation. IEEE Transactions on Intelligent Transportation Systems, 13(1), 284-295.

[3] Ferreira, M., Fernandes, R., Conceição, H., Viriyasitavat, W., \& Tonguz, O. K. (2010, September). Self-organized traffic control. In Proceedings of the seventh ACM international workshop on VehiculAr InterNETworking (pp. 85-90). ACM.

[4] Yapp, J., \& Kornecki, A. J. (2015, August). Safety analysis of virtual traffic lights. In 2015 20th International Conference on Methods and Models in Automation and Robotics (MMAR) (pp. 505-510). IEEE.

[5] Teo, K. T. K., Kow, W. Y., \& Chin, Y. K. (2010, September). Optimization of traffic flow within an urban traffic light intersection with genetic algorithm. In 2010 Second International Conference on Computational Intelligence, Modelling and Simulation (pp. 172177).

[6] Jun, M., \& Ying, M. (2008, December). Research of traffic flow forecasting based on neural network. In 2008 Second International Symposium on Intelligent Information Technology Application (Vol. 2, pp. 104-108). IEEE.

[7] Fazli, S., Mohammadi, S., \& Rahmani, M. (2012). Neural Network based vehicle classification for intelligent traffic control. International Journal of Software Engineering \& Applications, 3(3), 17.

[8] Chao, K. H., Lee, R. H., \& Wang, M. H. (2008, September). An intelligent traffic light control based on extension neural network. In International Conference on Knowledge-Based and Intelligent Information and Engineering Systems (pp. 17-24). Springer, Berlin, Heidelberg.

[9] Viswanathan, V., \& Santhanam, V. (2013). Intelligent traffic signal control using wireless sensor networks. In Proceding of 2nd International Conference on Advances in Electrical and Electronics

[10] Viraktamath, S. V., Holkar, P., Narayankar, P. V., \& Pujari, J. (2015). Adaptive Intelligent Traffic Control System using PLC. International Journal of Innovative Research in Computer and Communication Engineering, 287-290.

[11] D. (2019). What is fuzzy logic? - Definition from WhatIs.com. [online] SearchEnterpriseAI. Available at: https://searchenterpriseai.techtarget.com/definition/fuzzy-logic.

[12] GeeksforGeeks. (2018). Fuzzy Logic | Introduction. [online] Available at: https://www.geeksforgeeks.org/fuzzy-logic-introduction/.

[13] Omina, J. A. (2015). An intelligent traffic light control system based on fuzzy logic algorithm. International Academic Journal of Information Systems and Technology, 1(5), 1-17.

[14] Wang, J., Shao, Y., Ge, Y., \& Yu, R. (2019). A surof vehicle to everything (v2x) testing. Sensors, 19(2), 334.

[15] users.ece.cmu.edu. (n.d.). Virtual Traffic Lights. [online] Available at: https://users.ece.cmu.edu/ tonguz/vtl/about.html [Accessed 11 Mar. 2020]. 
[16] RCR Wireless News. (2020). Congressmen applaud FCC move to reallocate $5.9 \mathrm{GHz}$ for C-V2X. [online] Available at: https://www.rcrwireless.com/20200114/policy/congressmen-applaud-fcc-c-v2x\#prettyPhoto [Accessed 11 Mar. 2020].

[17] Anon, (n.d.). Technology - Virtual Traffic Lights. [online] Available at: https://www.virtualtrafficlights.com/technology/ [Accessed 11 Mar. 2020].

[18] Zhang, R., Schmutz, F., Gerard, K., Pomini, A., Basseto, L., Hassen, S. B., ... \& Tonguz, O. (2018, August). Virtual traffic lights: System design and implementation. In 2018 IEEE 88th Vehicular Technology Conference (VTC-Fall) (pp. 1-5). IEEE.

[19] Zhang, R., Jacquemot, B., Bakirci, K., Bartholme, S., Kaempf, K., Freydt, B., ... \& Tonguz, O. (2019). Leader selection in Vehicular Ad-hoc Network: a Proactive Approach. arXiv preprint arXiv:1912.06776.

[20] Bazzi, A., Zanella, A., \& Masini, B. M. (2016). A distributed virtual traffic light algorithm exploiting short range V2V communications. Ad Hoc Networks, 49, $42-57$.

[21] Ferreira, M., Fernandes, R., Conceição, H., Viriyasitavat, W., \& Tonguz, O. K. (2010, September). Self-organized traffic control. In Proceedings of the seventh ACM international workshop on VehiculAr InterNETworking (pp. 85-90).

[22] Münst, W., Dannheim, C., Mäder, M., Gay, N., Malnar, B., Al-Mamun, M., \& Icking, C. (2015, October). Virtual traffic lights: Managing intersections in the cloud. In 2015 7th International Workshop on Reliable Networks Design and Modeling (RNDM) (pp. 329-334). IEEE.

[23] Al-qutwani, M., \& Wang, X. (2019). Smart Traffic Lights over Vehicular Named Data Networking. Information, 10(3), 83. 